\title{
Wickerhamomyces anomalus: A Rare Fungal Sepsis in Neonates
}

\author{
Shantanu Shubham ${ }^{1} \cdot$ Shaik Naseeruddin $^{2} \cdot$ U. Sasi Rekha ${ }^{3} \cdot$ Mayank Priyadarshi $^{1}$ (D) $\cdot$ Pratima Gupta $^{3} \cdot$ Sriparna Basu $^{1}$
}

Received: 21 March 2021 / Accepted: 22 April 2021 / Published online: 20 May 2021

(C) Dr. K C Chaudhuri Foundation 2021

To the Editor: Recently, we came across two cases of rare invasive fungal sepsis in our neonatal unit caused by Wickerhamomyces anomalus (WA).

The first case was an outborn full-term $2.2 \mathrm{~kg}$ female neonate, referred to us on day 4 of life with worsening respiratory distress since $2 \mathrm{~h}$ after delivery. The neonate required mechanical ventilation (MV) with other supportive management. Blood culture through BACTEC system grew fungal colonies on Sabouraud agar. Matrix-assisted laser desorption ionizationtime of flight mass spectrometry (MALDI-TOF MS) used for species identification confirmed the growth of WA.

The second case was a late-preterm male weighing $2 \mathrm{~kg}$, referred to us at $20 \mathrm{~h}$ of life for respiratory distress noted soon after birth. The neonate required MV, surfactant administration, and inotropic support. Blood culture showed similar fungal growth, which, on MALDI-TOF, showed the same species of WA.

Both the cases were outborn, normally delivered, lowbirth-weight neonates with uneventful antenatal and perinatal history. Initial presentation was respiratory distress, which progressively increased in severity requiring MV. Sepsis screen was negative. Cerebrospinal fluid examination was normal, and culture was sterile. Both neonates recovered uneventfully after $14 \mathrm{~d}$ therapy with amphotericin-B.

WA is an ascomycetes yeast- $\mathrm{a}$ free-living form in plants, soil, and other organic matters [1]. It can multiply in varied

Mayank Priyadarshi

priyadarshi.aiims@gmail.com

1 Department of Neonatology, All India Institute of Medical Sciences, Rishikesh, Uttarakhand 249203, India

2 Department of Pediatrics, All India Institute of Medical Sciences, Rishikesh, Uttarakhand, India

3 Department of Microbiology, All India Institute of Medical Sciences, Rishikesh, Uttarakhand, India and extreme conditions, being resistant to extreme $\mathrm{pH}$, high osmotic pressure, and anaerobic conditions [2]. The clinical presentation of WA can be varied, such as apnea, thrombocytopenia, feeding intolerance, necrotizing enterocolitis, multiorgan failure, and even death [3]. The species is usually sensitive to common antifungals like fluconazole and amphotericin-B [4].

As both the infants were outborn, we could not specify the exact source of infection. Inappropriate asepsis principles could have been the causative factors. Timely diagnosis and intervention with amphotericin-B and other supportive management helped the neonates to make uneventful recovery.

\section{Declarations}

Consent to Publish The legal guardians have consented regarding publishing the data.

Conflict of Interest None.

\section{References}

1. Fredlund E, Druvefors U, Boysen ME, Lingsten KJ, Schnürer J. Physiological characteristics of the biocontrol yeast Pichia anomala J121. FEMS Yeast Res. 2002;2:395-402.

2. Murphy N, Buchanan CR, Damjanovic V, Whitaker R, Hart CA, Cooke RW. Infection and colonisation of neonates by Hansenula anomala. Lancet. 1986;1:291-3.

3. Marwah P, Kumar S, Marwah A. Pichia anomala a rare cause of nosocomial fungal sepsis in newborn. Is empirical use of third generation cephalosporin to blame? J Clin Diag Res. 2019;136:SD01-2.

4. Paula CR, Krebs VL, Auler ME, et al. Nosocomial infection in newborns by Pichia anomala in a Brazilian intensive care unit. Med Mycol. 2006;44:479-84.

Publisher's Note Springer Nature remains neutral with regard to jurisdictional claims in published maps and institutional affiliations. 